\title{
Structures of Layered Materials After Reaction with Li/Na
}

Manish Singh $^{1}$, Chanchal Ghosh ${ }^{1}$, Matthew Janish ${ }^{1}$, Shayani Parida ${ }^{1}$, Avinash M Dongare ${ }^{1}$ and C Barry Carter $^{1,2}$

${ }^{1}$ University of Connecticut, Storrs, Connecticut, United States, ${ }^{2}$ Center of Integrated Nanotechnologies, Sandia National Laboratories, Albuquerque, New Mexico, United States

Understanding structural changes that occur at the atomic scale of the layered materials during reaction of $\mathrm{Li} / \mathrm{Na}$ may help improve the performance of solid-state batteries using these materials [1-2]. New strategies and novel experimental designs are catalyzed by the availability of TEM holders for in-situ investigations. There are a growing number of reports pertaining to reactions of $\mathrm{Li}$ and $\mathrm{Na}$ with wide variety of materials ranging from transition metal dichalcogenides (TMDCs) to TM oxides (TMOs) both from bulk exfoliated flakes as well as chemically synthesized nanostructures. Qualitative features have been invariably shown in terms of change in contrast during reaction with $\mathrm{Li}$, which may be a signature of insertion of Li into TMDCs/TMOs [3]. However, The viewing direction can complicate the issue in particular for reactions with nanostructures with a range of possible orientations in addition to the challenges of performing the in-situ reactions inside TEM itself [4]. There are limited reports from structural viewpoint demonstrating the changes in the layered materials with the insertion of Li/Na.

In this paper, we report a study of reactions between $\mathrm{Li} / \mathrm{Na}$ with mechanically exfoliated flakes and powders of $\mathrm{WS}_{2}$ inside a FEI Tecnai F-30 TEM in-situ employing Nanofactory TEM- STM holder. The structural characterization of pre- and post-reacted specimens have been performed using SAD and HRTEM. The chemistry of the post-reacted $\mathrm{WS}_{2}$ after $\mathrm{Li} / \mathrm{Na}$ reaction has been studied using STEM-EELS and STEM-XEDS. The HRTEM images of the post-lithiated materials have been acquired using a Cscorrected Titan ETEM. These results are compared with experimental images obtained using $\mathrm{MoS}_{2}$, and initial computer modeling using DFT calculations for both $\mathrm{Li}$ and $\mathrm{Na}$ reactions.

Figure 1 shows the BF TEM images and electron diffraction patterns of reacted $\mathrm{WS}_{2}$ powders. The prereacted $\mathrm{WS}_{2}$ was indexed to be $2 \mathrm{H}-\mathrm{WS}_{2}$ along zone axis [0001]. Several extra reflections are observed apart from the $2 \mathrm{H}-\mathrm{WS}_{2}$ after $\mathrm{Li} / \mathrm{Na}$ reactions clearly suggests the formation of new phases. This interpretation is supported by high-resolution phase-contrast images from the same specimen. The same region of the reacted specimen has been examined for chemical and structural changes. The STEM-EELS line profiles of $\mathrm{Li} / \mathrm{Na}, \mathrm{W}$, and $\mathrm{S}$ along the reaction zone show undulations in the stoichiometry of $\mathrm{W}$ and $\mathrm{S}$ and in the content of $\mathrm{Li} / \mathrm{Na}$. This finding is also corroborated with HRTEM images coupled with image simulations using JEMS [5]. These results will also be compared with the structural changes in other layered materials, in particular $\mathrm{MoS}_{2}$ and graphite.

This research is funded by NSF under award DMR-1820565. The TEM investigation was carried out at CINT, an Office of Science User Facility operated for the U.S. DOE. Sandia National Labs is managed and operated by National Technology and Engineering Solutions of Sandia, LLC., a wholly owned subsidiary of Honeywell International, Inc., for the U.S. DOE's NNSA contract DE-NA-0003525. The views expressed here do not necessarily represent the views of the U.S. DOE or the U.S. Government. Matt Janish is presently a staff scientist at Los Alamos NL. 


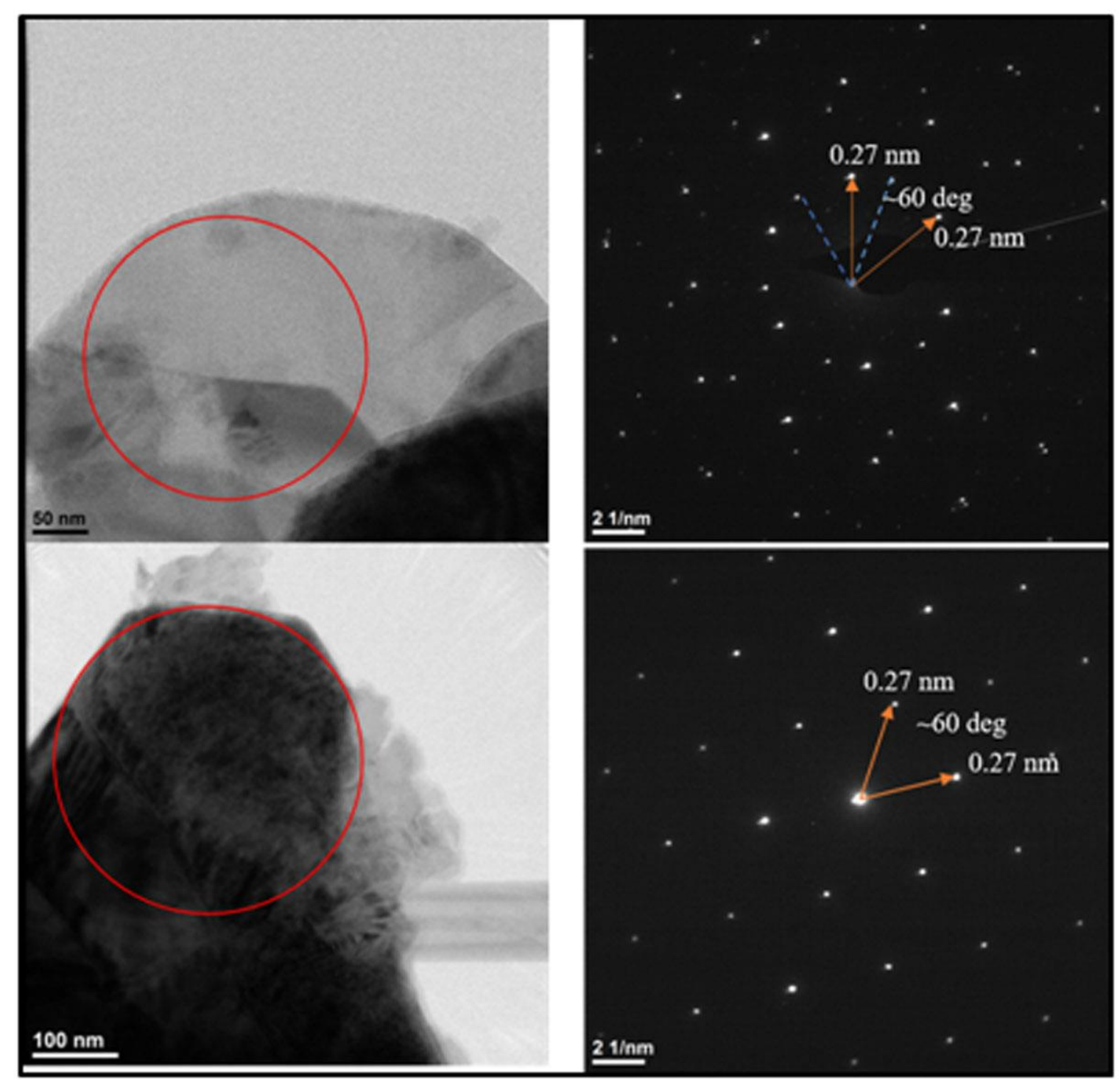

Figure 1. BF TEM images and corresponding electron diffraction patterns of reacted WS2 powder sample.

References [1] Larson DT, Fampiou I, Kim G, Kaxiras E (2018) Lithium intercalation in Graphene-MoS2 heterostructures, J. Phys. Chem. C 122, 24535-24541. [2] Cheng Y, Nie A, Zhang Q et al., (2014) Origin of phase transition in lithiated Molybdenum Disulphide, ACS Nano 8(11), 11447-11453. [3] Caputo R, Tekin A (2019) Can lithium form phases with molybdenum? J. Solid state Chemistry 271, 230-238. [4] Carter CB, Williams DB (Eds.) (2016) Transmission electron microscopy: diffraction, imaging and spectrometry, Springer, Berlin Heidelberg, 17-80 [5] Stadelmann P (1987) EMS - a software package for electron diffraction analysis and HREM image simulation in materials science, Ultramicroscopy 21(2), $131-145$ 